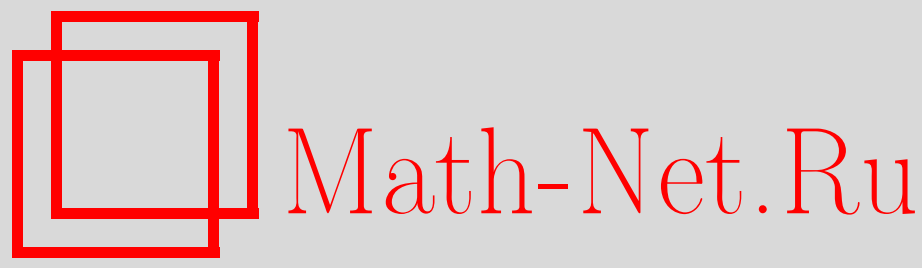

В. В. Жаринов, О гамильтоновых операторах в дифференциальных алгебрах, ТMФ, 2017, том 193, номер 3, 369-380

DOI: https://doi.org/10.4213/tmf9400

Использование Общероссийского математического портала Math-Net.Ru подразумевает, что вы прочитали и согласны с пользовательским соглашением http://www . mathnet.ru/rus/agreement

Параметры загрузки:

IP: 35.173 .219 .12

26 апреля 2023 г., 10:47:17

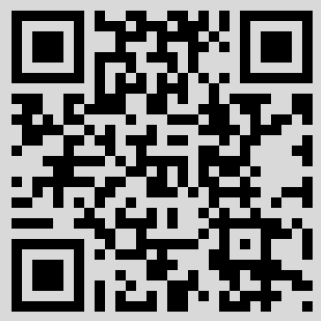




\title{
ФИЗИКА
}

Том 193, № 3

декабрь, 2017

(C) 2017 г.

В. В. Жаринов*

\section{О ГАМИЛЬТОНОВЫХ ОПЕРАТОРАХ В ДИФФЕРЕНЦИАЛЬНЫХ АЛГЕБРАХ}

\begin{abstract}
Развивается предложенная ранее алгебраическая техника для гамильтонова подхода к эволюционным системам уравнений в частных производных, включая системы со связями, и предлагается определяющая система уравнений (пригодная для компьютерных вычислений), характеризующая гамильтоновы операторы данного вида. Эта техника демонстрируется на простом примере.
\end{abstract}

Ключевые слова: дифференциальная алгебра, структура Ли-Пуассона, тождество Якоби, гамильтонов оператор, гамильтонова эволюционная система.

DOI: https://doi.org/10.4213/tmf9400

\section{1. ВВЕДЕНИЕ}

В предыдущей работе [1] мы предложили алгебраическую технику (основанную на книге [2], глава VII) для гамильтонова подхода к эволюционным системам уравнений в частных производных, включая системы со связями. Здесь мы развиваем этот подход и предлагаем определяющую систему уравнений (пригодную для компьютерных вычислений), характеризующую гамильтоновы операторы данного вида.

Мы работаем в рамках алгебро-геометрического подхода к уравнениям в частных производных (см., например, [2]-[4]). Мы надеемся, что наши результаты окажутся полезными в исследованиях, связанных с работами [5]--[19].

Мы используем следующие общие обозначения:

- $\mathbb{F}=\mathbb{R}, \mathbb{C}, \quad \mathbb{N}=\{1,2,3, \ldots\} \subset \mathbb{Z}_{+}=\{0,1,2, \ldots\} ;$

- $\mathrm{M}=\{1, \ldots, m\}, \quad m \in \mathbb{N}$;

- $\mathbb{I}=\mathbb{Z}_{+}^{\mathrm{M}}=\left\{i=\left(i^{1}, \ldots, i^{m}\right) \mid i^{\mu} \in \mathbb{Z}_{+}, \mu \in \mathrm{M}\right\}$.

Все линейные операции выполняются над числовым полем $\mathbb{F}$. Как правило, предполагается суммирование по повторяющимся верхним и нижним индексам.

Исследование выполнено за счет гранта Российского научного фонда (проект № 14-50-00005).

* Математический институт им. В. А. Стеклова Российской академии наук, Москва, Россия. E-mail: zharinov@mi.ras.ru 


\section{2. ДИФФЕРЕНЦИАЛЬНАЯ АЛГЕБРА}

Основной объект в нашей конструкции - дифференциальная алгебра $\left(\mathcal{F}, \mathfrak{D}_{H}\right)$, где (детали и мотивировки см. в [1]):

- $\mathcal{F}$ - унитальная (т. е. содержащая единичный элемент) ассоциативная коммутативная алгебра;

- $\mathfrak{D}=\mathfrak{D}(\mathcal{F})$ - алгебра Ли всех дифференцирований алгебры $\mathcal{F} ;$ предполагается, что $\mathfrak{D}=\mathfrak{D}_{V} \oplus_{\mathcal{F}} \mathfrak{D}_{H}$

- $\mathfrak{D}_{V}=\mathfrak{D}_{V}(\mathcal{F})$ - вертикальная подалгебра алгебры Ли $\mathfrak{D}$ с формальным $\mathcal{F}$-базисом $\partial=\left\{\partial_{a} \mid a \in \mathbf{A}\right\},\left[\partial_{a}, \partial_{b}\right]=0, a, b \in \mathbf{A}, \mathbf{A}$ - множество индексов, в частности $\mathfrak{D}_{V}=\left\{X=X^{a} \partial_{a} \mid X^{a} \in \mathcal{F}\right\}$, где для любого элемента $L \in \mathcal{F}$ действие $\partial_{a} L \neq 0$ только для конечного числа индексов $a \in \mathbf{A}$;

- $\mathfrak{D}_{H}=\mathfrak{D}_{H}(\mathcal{F})$ - горизонтальная подалгебра алгебры Ли $\mathfrak{D}$ с $\mathcal{F}$-базисом $D=$ $\left\{D_{\mu} \mid \mu \in \mathrm{M}\right\},\left[D_{\mu}, D_{\nu}\right]=0, \mu, \nu \in \mathrm{M}$

- $\left[D, \mathfrak{D}_{V}\right] \subset \mathfrak{D}_{V}$, т. е. $\left[D_{\mu}, V\right] \in \mathfrak{D}_{V}$ для всех $\mu \in \mathrm{M}$ и $V \in \mathfrak{D}_{V}$.

Напомним, что множество $\mathfrak{D}$ обладает двумя алгебраическими структурами: структурой алгебры Ли с коммутатором $[X, Y]=X \circ Y-Y \circ X$ в качестве скобки Ли и структурой $\mathcal{F}$-модуля с умножением $(K \cdot X) L=K \cdot(X L)$, которые связаны условием согласования $[X, L \cdot Y]=(X L) \cdot Y+L \cdot[X, Y]$ для всех $K, L \in \mathcal{F}$ и для всех $X, Y \in \mathfrak{D}$.

Для любых двух множеств индексов $\mathbf{A}, \mathbf{B}$ множество $\mathcal{F}_{\mathbf{B}}^{\mathbf{A}}=\left\{\boldsymbol{\eta}=\left(\eta_{b}^{a}\right) \mid \eta_{b}^{a} \in \mathcal{F}\right.$, $a \in \mathbf{A}, b \in \mathbf{B}\}$ обладает структурой $\mathcal{F}$-модуля с покомпонентным умножением, т. е. $K \cdot \boldsymbol{\eta}=\left(K \cdot \eta_{b}^{a}\right) \in \mathcal{F}_{\mathbf{B}}^{\mathbf{A}}$ для всех $K \in \mathcal{F}, \eta \in \mathcal{F}_{\mathbf{B}}^{\mathbf{A}}$.

Через $\stackrel{\circ}{\mathcal{F}}_{\mathbf{B}}^{\mathbf{A}}$ мы обозначим $\mathcal{F}$-модуль всех элементов $\eta=\left(\eta_{b}^{a}\right) \in \mathcal{F}_{\mathbf{B}}^{\mathbf{A}}$ таких, что для любого верхнего индекса $a \in \mathbf{A}$ только конечное число компонент $\eta_{b}^{a} \neq 0$. В частности, $\stackrel{\circ}{\mathcal{F}}^{\mathbf{A}}=\mathcal{F}^{\mathbf{A}}$ для любого множества индексов $\mathbf{A}$, тогда как $\stackrel{\circ}{\mathcal{F}}_{\mathbf{B}}$ состоит из всех конечных элементов $\eta=\left(\eta_{b}\right) \in \mathcal{F}_{\mathbf{B}}$, и $\stackrel{\circ}{\mathcal{F}}_{\mathbf{B}}=\mathcal{F}_{\mathbf{B}}$ тогда и только тогда, когда множество индексов В конечное. Например, согласно приведенному выше соглашению $\partial K=\left(\partial_{a} K\right) \in \stackrel{\circ}{\mathcal{F}}_{\mathbf{A}}$ для любого элемента $K \in \mathcal{F}$, в частности определено линейное отображение $\partial: \mathcal{F} \rightarrow \stackrel{\circ}{\mathcal{F}}_{\mathbf{A}}$.

Для любого $\mathcal{F}$-модуля $\mathcal{F}_{\mathbf{B}}^{\mathbf{A}}$ и для любого дифференцирования $X \in \mathfrak{D}$ определено покомпонентное линейное отображение $X: \mathcal{F}_{\mathbf{B}}^{\mathbf{A}} \rightarrow \mathcal{F}_{\mathbf{B}}^{\mathbf{A}}, \eta=\left(\eta_{b}^{a}\right) \mapsto X \eta=\left(X \eta_{b}^{a}\right)$. В частности, правило Лейбница имеет вид $X(K \cdot \eta)=(X K) \cdot \boldsymbol{\eta}+K \cdot(X \eta)$ для всех $X \in \mathfrak{D}, K \in \mathcal{F}, \eta \in \mathcal{F}_{\mathbf{B}}^{\mathbf{A}}$.

В этих обозначениях коммутатор $\left[D_{\mu}, \partial_{a}\right]=\Gamma_{\mu a}^{b} \cdot \partial_{b}$ для всех $\mu \in \mathrm{M}, a \in \mathbf{A}$, и мы предполагаем, что символ $\Gamma=\left(\Gamma_{\mu a}^{b}\right) \in \stackrel{\circ}{\mathcal{F}}_{\text {МА }}^{\mathbf{A}}$. Заметим, что здесь множество М конечное, тогда как множество $\mathbf{A}$, как правило, бесконечное, и согласно приведенному выше определению $\mathcal{F}$-модуля $\stackrel{\circ}{\mathcal{F}}_{\text {МА }}^{\mathbf{A}}$ для всякого индекса $b \in \mathbf{A}$ только конечное число компонент $\Gamma_{\mu a}^{b} \neq 0$.

\section{3. ГОРИЗОНТАЛЬНЫЕ ДИФФЕРЕНЦИАЛЬНЫЕ ОПЕРАТОРЫ}

Мы называем линейное отображение $P(D): \mathcal{F} \rightarrow \mathcal{F}\left(\right.$ т. е. $P(D) \in \operatorname{End}_{\mathbb{F}}(\mathcal{F})$ ) горизонтальным дифференииальным оператором, если $P(D)=P_{i} \cdot D^{i}$, где индексы $i=\left(i^{1}, \ldots, i^{m}\right) \in \mathbb{I}$, коэффициенты $P=\left(P_{i}\right) \in \stackrel{\circ}{\mathcal{F}}_{\mathbb{I}}$, дифференциальные мономы 
$D^{i}=\left(D_{1}\right)^{i^{1}}$ 。.。 $\left(D_{m}\right)^{i^{m}}$, так что $P(D) L=P_{i} \cdot D^{i} L$ для всех $L \in \mathcal{F}$. Множество всех горизонтальных дифференциальных операторов мы обозначим через $\mathcal{F}[D]$. Это унитальная ассоциативная алгебра с умножением, задаваемым правилом композииии отображений. Аналогичным образом для любых четырех множеств индексов $\mathbf{A}, \mathbf{B}, \mathbf{A}^{\prime}, \mathbf{B}^{\prime}$ определен $\mathcal{F}[D]$-модуль $\mathcal{F}_{\mathbf{B}^{\prime} \mathbf{A}}^{\mathbf{A}^{\prime} \mathbf{B}}[D]$ матричных горизонтальных дифференциальных операторов

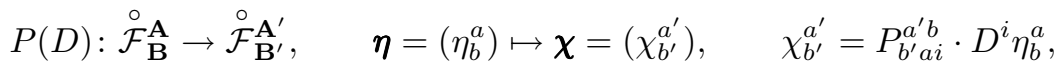

где $P=\left(P_{b^{\prime} a i}^{a^{\prime} b}\right) \in \stackrel{\circ}{\mathcal{F}_{\mathbf{B}^{\prime}}^{\mathbf{A}^{\prime} \mathbf{B} \mathbb{I}}}, P(D)=\left(P_{b^{\prime} a i}^{a^{\prime} b} \cdot D^{i}\right)$.

Для каждой пары множеств индексов $\mathbf{A}, \mathbf{B}$ определено естественное спаривание

$$
\langle\cdot, \cdot\rangle: \stackrel{\circ}{\mathcal{F}}_{\mathbf{B}}^{\mathbf{A}} \times \stackrel{\circ}{\mathcal{F}}_{\mathbf{A}}^{\mathbf{B}} \rightarrow \mathcal{F}, \quad \eta=\left(\eta_{b}^{a}\right), \quad \boldsymbol{\zeta}=\left(\zeta_{a}^{b}\right) \mapsto\langle\boldsymbol{\eta}, \boldsymbol{\zeta}\rangle=\eta_{b}^{a} \cdot \zeta_{a}^{b}
$$

Для каждого горизонтального дифференциального оператора $P(D): \stackrel{\circ}{\mathcal{F}} \mathbf{\mathbf { A }} \rightarrow \stackrel{\circ}{\mathcal{F}_{\mathbf{B}^{\prime}}^{\prime}}$ определены сопряженный по Лагранжу оператор

$$
\stackrel{*}{P}(D): \stackrel{\circ}{\mathcal{F}}{\mathbf{\mathbf { A } ^ { \prime }}}^{\mathbf{B}^{\prime}} \rightarrow \stackrel{\circ}{\mathcal{F}} \mathbf{\mathbf { B }}, \quad \boldsymbol{\zeta}=\left(\zeta_{a^{\prime}}^{b^{\prime}}\right) \mapsto \boldsymbol{\omega}=\left(\omega_{a}^{b}\right), \quad \omega_{a}^{b}=(-D)^{i}\left(\zeta_{a^{\prime}}^{b^{\prime}} \cdot P_{b^{\prime} a i}^{a^{\prime} b}\right),
$$

где $\stackrel{*}{P}=\left(\stackrel{*}{P} \underset{a b^{\prime} i}{b a^{\prime}}\right) \in \stackrel{\circ}{\mathcal{F}}{\stackrel{\mathbf{A A}}{\mathbf{B}^{\prime} \mathbb{I}}}^{\prime}, \stackrel{*}{P}(D)=\left(\stackrel{*}{P} \underset{a b^{\prime} i}{b a^{\prime}} \cdot D^{i}\right)=\left((-D)^{i} \circ P_{b^{\prime} a i}^{a^{\prime} b}\right)$, и бормула Грина (формула интегрирования по частям)

$$
\langle\boldsymbol{\zeta}, P(D) \boldsymbol{\eta}\rangle-\langle\stackrel{*}{P}(D) \boldsymbol{\zeta}, \boldsymbol{\eta}\rangle=D_{\mu} \psi^{\mu} \quad \text { для всех } \quad \boldsymbol{\zeta} \in \stackrel{\circ}{\mathcal{F}}_{\mathbf{A}^{\prime}}^{\mathbf{B}^{\prime}}, \boldsymbol{\eta} \in \stackrel{\circ}{\mathcal{F}}_{\mathbf{A}}^{\mathbf{B}}
$$

с некоторым током $\psi=\left(\psi^{\mu}(\boldsymbol{\zeta}, P(D), \boldsymbol{\eta})\right) \in \mathcal{F}^{\mathrm{M}}$.

ПредПОЛОЖЕНИЕ 1. Мы предполагаем, что дифберенциалъная алгебра $\left(\mathcal{F}, \mathfrak{D}_{H}\right)$ горизонтально точная, т. е. горизонтальный дибференциальный оператор $P(D)=$ $P_{i} \cdot D^{i}=0(P(D) K=0$ для всех $K \in \mathcal{F})$ тогда и только тогда, когда его коэффиииенты $P_{i}=0$ для всех $i \in \mathbb{I}$.

Заметим, что в этом случае таким же свойством обладают и матричные операторы.

\section{4. ОСНОВНЫЕ ИНГРЕДИЕНТЫ}

Основные компоненты, инструменты и предположения предлагаемой техники следующие (подробнее см. [1]):

• горизонтальный дифференциальный оператор

$$
D: \mathcal{F} \rightarrow \mathcal{F}_{\mathrm{M}}, \quad K \mapsto D K=\left(D_{\mu} K\right) ;
$$

• горизонтальный дифференциальный оператор

$$
\operatorname{Div}=-\stackrel{*}{D}: \mathcal{F}^{\mathrm{M}} \rightarrow \mathcal{F}, \quad \psi=\left(\psi^{\mu}\right) \mapsto \operatorname{Div} \psi=D_{\mu} \psi^{\mu}
$$

- горизонтальный дифференциальный оператор

$$
\nabla: \mathcal{F}^{\mathbf{A}} \rightarrow \mathcal{F}_{\mathrm{M}}^{\mathbf{A}}, \quad \phi=\left(\phi^{a}\right) \mapsto \eta=\left(\eta_{\mu}^{a}\right), \quad \eta_{\mu}^{a}=D_{\mu} \phi^{a}+\Gamma_{\mu b}^{a} \cdot \phi^{b} ;
$$


- сопряженный по Лагранжу оператор

$$
\stackrel{*}{\nabla}: \stackrel{\circ}{\stackrel{\mathcal{F}}{\mathrm{A}}_{\mathrm{M}}^{\mathrm{M}}} \rightarrow \stackrel{\circ}{\mathcal{F}} \mathbf{A}, \quad \chi=\left(\chi_{a}^{\mu}\right) \mapsto \mathbf{f}=\left(f_{a}\right), \quad f_{a}=-D_{\mu} \chi_{a}^{\mu}+\Gamma_{\mu a}^{b} \chi_{b}^{\mu}
$$

- вертикальный дифференииальный оператор

$$
\partial: \mathcal{F} \rightarrow \stackrel{\circ}{\mathcal{F}}_{\mathbf{A}}, \quad K \mapsto \partial K=\left(\partial_{a} K\right) .
$$

Оператор $\nabla$ корректно определен, в силу предположения $\Gamma=\left(\Gamma_{\mu a}^{b}\right) \in \stackrel{\circ}{\mathcal{F}} \mathbf{A}$ A

ПредЛОжение 1. Определенъ композиции $\partial \circ \mathrm{Div}, \stackrel{*}{\nabla} \circ \partial: \mathcal{F}^{\mathrm{M}} \rightarrow \stackrel{\circ}{\mathcal{F}} \mathbf{A}$, и справедливо равенство $\partial \circ \operatorname{Div}+\stackrel{*}{\nabla} \circ \partial=0$.

ДокАзАтельство. Действительно, для любого вектора $\psi=\left(\psi^{\mu}\right) \in \mathcal{F}^{\mathrm{M}}$ имеет место равенство

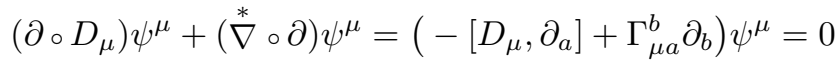

согласно определению символа Г.

Нам также понадобятся:

- линейное подпространство $\mathcal{E}=\operatorname{Ker} \nabla$ линейного пространства $\mathcal{F}^{\mathbf{A}}$;

- множество $\mathfrak{D}_{E}=\mathfrak{D}_{E}(\mathcal{F})=\left\{V \in \mathfrak{D}_{V} \mid\left[D_{\mu}, V\right]=0, \mu \in \mathrm{M}\right\}$ всех эволющионных дифференцирований алгебры $\mathcal{F}$.

Множество $\mathfrak{D}_{E}$ есть подалгебра алгебры Ли $\mathfrak{D}_{V}$, поскольку $\left[\mathfrak{D}_{E}, \mathfrak{D}_{E}\right] \subset \mathfrak{D}_{E}$, в силу тождества Якоби для коммутаторов, но не подмодуль $\mathcal{F}$-модуля $\mathfrak{D}_{V}$ (см., например, [3]).

ПреДЛОЖениЕ 2. Отображение еv: $\mathcal{E} \rightarrow \mathfrak{D}_{E}, \phi=\left(\phi^{a}\right) \mapsto \mathrm{ev}_{\phi}=\phi^{a} \cdot \partial_{a}$, есть изоморбизм линейных пространств. Более того, структура алгебры Ли на линейном пространстве $\mathfrak{D}_{E}$ задает изоморфную структуру на линейном пространстве $\mathcal{E}$ правилом

$$
[\phi, \psi]=\xi, \quad \phi=\left(\phi^{a}\right), \boldsymbol{\psi}=\left(\psi^{a}\right), \quad \xi=\left(\xi^{a}\right), \quad \xi^{a}=\operatorname{ev}_{\phi} \psi^{a}-\operatorname{ev}_{\boldsymbol{\psi}} \phi^{a}, \quad a \in \mathbf{A}
$$

ДокАЗАтЕльство. Доказательство осуществляется прямой проверкой.

ПрЕДЛОЖЕНИЕ 3. Для каждого горизонталъного дифференицального оператора $P(D)=\left(P_{i b}^{a} \cdot D^{i}\right): \mathcal{F}^{\mathbf{B}} \rightarrow \mathcal{F}^{\mathbf{A}}$, его сопряженного по Лагранжу $\stackrel{*}{P}(D)=\left((-D)^{i} \circ P_{i b}^{a}\right):$ $\stackrel{\circ}{\mathcal{F}}_{\mathbf{A}} \rightarrow \stackrel{\circ}{\mathcal{F}}_{\mathbf{B}}$ и любого $\phi \in \mathcal{E}$ справедливы следующие утверждения:

- $\left[\operatorname{ev}_{\boldsymbol{\phi}}, P(D)\right]=\operatorname{ev}_{\boldsymbol{\phi}} P(D)=\left(\left(\operatorname{ev}_{\boldsymbol{\phi}} P_{i b}^{a}\right) \cdot D^{i}\right)$;

- $\left[\mathrm{ev}_{\boldsymbol{\phi}}, \stackrel{*}{P}(D)\right]=\mathrm{ev}_{\boldsymbol{\phi}} \stackrel{*}{P}(D)=\left((-D)^{i} \circ\left(\mathrm{ev}_{\boldsymbol{\phi}} P_{i b}^{a}\right)\right)=\left[\mathrm{ev}_{\boldsymbol{\phi}}, P(D)\right]^{*}$;

m.е. эволюиионное дифберенцирование $\mathrm{ev}_{\phi}$ здесъ действует покомпонентно.

ДокАзАтЕльство. Доказательство опирается на характеристическое свойство эволюционных дифференцирований. 
ПРЕДПОЛОЖЕНИЕ 2. Мъ предполагаем, что существует множество индексов А и горизонталъный дифференциальный оператор $\mathbf{J}=J(D): \mathcal{F}^{\mathrm{A}} \rightarrow \mathcal{F}^{\mathbf{A}}, \phi=\left(\phi^{\alpha}\right) \mapsto$ $\phi=\left(\phi^{a}\right)$ maкой, чmо

а) композиция $\nabla \circ \mathbf{J}=0$, m.e. $\operatorname{Im} \mathbf{J} \subset \operatorname{Ker} \nabla$;

б) коммутатор $\left.\left[\mathrm{ev}_{\boldsymbol{\phi}}, \mathbf{J}\right)\right]=0$ для всех $\boldsymbol{\phi} \in \mathcal{E}$.

Подробнее, $\mathbf{J}=\left(J_{\alpha i}^{a} \cdot D^{i}\right), J=\left(J_{\alpha i}^{a}\right) \in \stackrel{\circ}{\mathcal{F}}_{\mathrm{AI}}^{\mathbf{A}}, \phi^{a}=J_{\alpha i}^{a} \cdot D^{i} \phi^{\alpha}$.

ПреДЛОЖенИЕ 4. Для сопряженного по Лагранжу оператора $\stackrel{*}{\mathbf{J}}: \stackrel{\circ}{\mathcal{F}}_{\mathbf{A}} \rightarrow \stackrel{\circ}{\mathcal{F}}_{\mathrm{A}}$ справедливы следующие утверждения:

а*) композичия $\stackrel{*}{\mathbf{J}} \circ \stackrel{*}{\nabla}=0$;

б*) коммутатор $\left[\mathrm{ev}_{\boldsymbol{\phi}}, \stackrel{*}{\mathbf{J}}\right]=0$ для всех $\boldsymbol{\phi} \in \mathcal{E}$.

ДокАЗАтЕльство. Доказательство осуществляется прямой проверкой.

\section{5. СТРУКТУРЫ ЛИ-ПУАССОНА}

Мы будем использовать следующие обозначения:

- $\mathfrak{F}=\mathcal{F} / \operatorname{Im} \operatorname{Div}=\left\{\int K=K+\operatorname{Div} \mathcal{F}^{\mathrm{M}} \mid K \in \mathcal{F}\right\}$;

- $\mathcal{E}=\mathcal{F}^{\mathrm{A}}, \mathcal{E}^{*}=\operatorname{Hom}_{\mathcal{F}}(\mathcal{E} ; \mathcal{F})=\stackrel{\circ}{\mathcal{F}}_{\mathrm{A}}$.

Здесь $\mathfrak{F}$ - линейное пространство всех функиионалов над линейным пространством $\mathcal{F}, \mathcal{E}$ - линейное пространство, а $\mathcal{E}^{*}$ - его дуальное пространство. Естественная проекция $\mathcal{F} \rightarrow \mathfrak{F}$ действует по правилу $K \mapsto \int K=K+\operatorname{Div} \mathcal{F}^{\mathrm{M}}$.

ПреДПОЛОжЕНИЕ 3. Мы предполагаем, что дифференииальная алгебра $\left(\mathcal{F}, \mathfrak{D}_{H}\right)$ имеет тип Дюбуа-Реймона, т.е. обладает следующим свойством: для данного элемента $K \in \mathcal{F}$ равенство $\int K \cdot L=0$ выполняется для всех $L \in \mathcal{F}$ тогда и только тогда, когда $K=0$.

В силу предложений 1 и 4 справедливы следующие утверждения: $\partial: \operatorname{Im} \operatorname{Div} \rightarrow$ $\operatorname{Im} \stackrel{*}{\nabla}$ и $\stackrel{*}{\mathbf{J}} \circ \stackrel{*}{\nabla}=0$, следовательно, определена последовательность линейных пространств

$$
\mathcal{F} / \operatorname{Im} \operatorname{Div} \stackrel{\partial}{\longrightarrow} \stackrel{\circ}{\mathcal{F}}_{\mathbf{A}} / \operatorname{Im} \stackrel{*}{\nabla} \stackrel{\text { J }}{\longrightarrow} \stackrel{\circ}{\mathcal{F}}_{\mathrm{A}}
$$

В частности, определено линейное отображение (вариационная производная)

$$
\text { • } \delta=\stackrel{*}{\mathbf{J}} \circ \partial: \mathfrak{F} \rightarrow \mathcal{E}^{*}, \quad \int K \mapsto \delta K=\stackrel{*}{\mathbf{J}}(\partial K) .
$$

ОПРЕДЕЛЕНиЕ 1. Структура Ли-Пуассона над дифференциальной алгеброй $\left(\mathcal{F}, \mathfrak{D}_{H}\right)$ есть билинейное отображение (скобка Ли-Пуассона)

$$
\{\cdot, \cdot\}: \mathfrak{F} \times \mathfrak{F} \rightarrow \mathfrak{F}, \quad \int K, \int L \mapsto\left\{\int K, \int L\right\},
$$

со следующими свойствами:

- $\left\{\int K, \int L\right\}+\left\{\int L, \int K\right\}=0$;

- $\mathbf{J I}\left(\int K, \int L, \int M\right)=\left\{\int K,\left\{\int L, \int M\right\}\right\}+$ c.p. $=0 ; \quad$ (тождество Якоби)

где аббревиатура "c.p." означает циклическую перестановку аргументов $\int K, \int L$, $\int M \in \mathfrak{F}$. В этом случае пара $(\mathfrak{F},\{\cdot, \cdot\})$ есть алгебра Ли. 
ОПРЕДЕЛЕНиЕ 2. Мы зададим скобку $\{\cdot, \cdot\}$ правилом

$$
\left\{\int K, \int L\right\}=\int\langle\delta K, \Lambda(D) \delta L\rangle \quad \text { для всех } \quad \int K, \int L \in \mathfrak{F},
$$

где горизонтальный дифференциальный оператор

$$
\Lambda(D): \mathcal{E}^{*} \rightarrow \mathcal{E}, \quad f=\left(f_{\alpha}\right) \mapsto \phi=\left(\phi^{\alpha}\right), \quad \phi^{\alpha}=\Lambda_{i}^{\alpha \beta} \cdot D^{i} f_{\beta}, \quad \alpha \in \mathrm{A},
$$

множество коэффициентов $\Lambda=\left(\Lambda_{i}^{\alpha \beta}\right) \in \stackrel{\circ}{\mathcal{F}}_{\mathbb{I}}^{\mathrm{AA}}$. Мы считаем, что оператор $\Lambda(D)$ кососимметрический по Лагранжу, т. е. $\Lambda(D)+\stackrel{*}{\Lambda}(D)=0$.

В этом случае скобка $\{\cdot, \cdot\}$ кососимметрическая, и наша задача сводится к тому, чтобы найти по возможности простое и эффективное условие гамильтоновости оператора $\Lambda(D)$, т. е. обеспечить выполнение тождества Якоби.

В статье [1] мы доказали следующий алгебраический аналог теоремы 7.8 из книги [2].

Лемма 1. Тождество Якоби имеет следующее представление:

$$
\mathbf{J I}\left(\int K, \int L, \int M\right)=\int\left\langle\delta K,\left[\operatorname{ev}_{\boldsymbol{\phi}(L)}, \Lambda(D)\right] \delta M\right\rangle+\text { c.p. }=0,
$$

где $\phi(L)=(\mathbf{J} \circ \Lambda(D) \circ \delta) L, \Lambda(D)=\left(\Lambda_{i}^{\alpha \beta} \cdot D^{i}\right)$ - горизонтальный дифференциалъный кососимметрический по Лагранжу оператор.

Здесь мы усилим этот результат и придадим ему вид, более удобный для реальных вычислений.

ОПРЕДЕЛЕНИЕ 3. Будем говорить, что тождество Якоби выполняется в усиленной форме, если

$$
\int\left\langle f,\left[\operatorname{ev}_{\phi(g)}, \Lambda(D)\right] h\right\rangle+\text { c.p. }=0
$$

для всех $f, g, h \in \mathcal{E}^{*}$, где $\phi(g)=(\mathbf{J} \circ \Lambda(D)) g$.

ЛЕмма 2. В условиях леммы 1 тождество Якоби в усиленной форме может быть записано в виде

$$
R_{k l}^{\alpha \beta \gamma} \cdot D^{k} g_{\beta} \cdot D^{l} h_{\gamma}+(-D)^{l}\left(R_{k l}^{\beta \gamma \alpha} \cdot g_{\beta} \cdot D^{k} h_{\gamma}\right)+(-D)^{k}\left(R_{k l}^{\gamma \alpha \beta} \cdot D^{l} g_{\beta} \cdot h_{\gamma}\right)=0
$$

для всех $g, h \in \mathcal{E}^{*}, \alpha, \beta, \gamma \in \mathrm{A}, k, l \in \mathbb{I}$, где коэфбициенты

$$
R_{k l}^{\alpha \beta \gamma}=R_{k l}^{\alpha \beta \gamma}(\Lambda)=\left(\begin{array}{c}
i \\
r
\end{array}\right) J_{\epsilon i}^{a} \cdot D^{i-r} \Lambda_{k-r}^{\epsilon \beta} \cdot \partial_{a} \Lambda_{l}^{\alpha \gamma} .
$$

ДокАЗАтЕЛЬСтво. Нам необходимо уточнить выражение

$$
\int\left\langle f,\left[\operatorname{ev}_{\boldsymbol{\phi}(g)}, \Lambda(D)\right] h\right\rangle=\int f_{\alpha} \cdot\left[\operatorname{ev}_{\phi(g)}, \Lambda_{l}^{\alpha \gamma} \cdot D^{l}\right] h_{\gamma},
$$

где $f, g, h \in \mathcal{E}^{*}$. Здесь $\phi(g)=(\mathbf{J} \circ \Lambda(D)) g$, т. е.

$$
\begin{aligned}
\phi^{a}(g) & =J_{\epsilon i}^{a} \cdot D^{i}\left(\Lambda_{k}^{\epsilon \beta} \cdot D^{k} g_{\beta}\right)=J_{\epsilon i}^{a} \cdot\left(\begin{array}{c}
i \\
r
\end{array}\right) \cdot D^{i-r} \Lambda_{k}^{\epsilon \beta} \cdot D^{k+r} g_{\beta}= \\
& =\left(\begin{array}{c}
i \\
r
\end{array}\right) \cdot J_{\epsilon i}^{a} \cdot D^{i-r} \Lambda_{k-r}^{\epsilon \beta} \cdot D^{k} g_{\beta} .
\end{aligned}
$$


Следовательно, согласно предложению 3

$$
\begin{aligned}
\int f_{\alpha} \cdot\left[\mathrm{ev}_{\boldsymbol{\phi}(g)}, \Lambda_{l}^{\alpha \gamma} \cdot D^{l}\right] h_{\gamma} & =\int f_{\alpha} \cdot\left(\begin{array}{l}
i \\
r
\end{array}\right) \cdot J_{\epsilon i}^{a} \cdot D^{i-r} \Lambda_{k-r}^{\epsilon \beta} \cdot D^{k} g_{\beta} \cdot \partial_{a} \Lambda_{l}^{\alpha \gamma} \cdot D^{l} h_{\gamma}= \\
& =\int R_{k l}^{\alpha \beta \gamma} \cdot f_{\alpha} \cdot D^{k} g_{\beta} \cdot D^{l} h_{\gamma} .
\end{aligned}
$$

Таким образом,

$$
\begin{aligned}
& \int\left\langle\delta K,\left[\operatorname{ev}_{\phi(L)}, \Lambda(D)\right] \delta M\right\rangle+\text { c.p. }= \\
& =\int R_{k l}^{\alpha \beta \gamma} \cdot\left(f_{\alpha} \cdot D^{k} g_{\beta} \cdot D^{l} h_{\gamma}+g_{\alpha} \cdot D^{k} h_{\beta} \cdot D^{l} f_{\gamma}+h_{\alpha} \cdot D^{k} f_{\beta} \cdot D^{l} g_{\gamma}\right)= \\
& =\int\left(R_{k l}^{\alpha \beta \gamma} \cdot f_{\alpha} \cdot D^{k} g_{\beta} \cdot D^{l} h_{\gamma}+R_{k l}^{\beta \gamma \alpha} \cdot D^{l} f_{\alpha} \cdot g_{\beta} \cdot D^{k} h_{\gamma}+R_{k l}^{\gamma \alpha \beta} \cdot D^{k} f_{\alpha} \cdot D^{l} g_{\beta} \cdot h_{\gamma}\right)= \\
& =\int f_{\alpha} \cdot\left(R_{k l}^{\alpha \beta \gamma} \cdot D^{k} g_{\beta} \cdot D^{l} h_{\gamma}+(-D)^{l}\left(R_{k l}^{\beta \gamma \alpha} \cdot g_{\beta} \cdot D^{k} h_{\gamma}\right)+(-D)^{k}\left(R_{k l}^{\gamma \alpha \beta} \cdot D^{l} g_{\beta} \cdot h_{\gamma}\right)\right),
\end{aligned}
$$

где мы дважды воспользовались формулой Грина интегрирования по частям. Ясно, что последнее представление и предполагаемое свойство Дюбуа-Реймона дифференциальной алгебры $\left(\mathcal{F}, \mathfrak{D}_{H}\right)$ влекут наше утверждение.

Теорема 1. В условиях леммы 2 тождество Якоби в усиленной форме сводится к системе уравнений $Q=Q(\Lambda)=\left(Q_{k l}^{\alpha \beta \gamma}\right)=0$, или, более подробно,

$$
Q_{k l}^{\alpha \beta \gamma}=0 \quad \text { дляввех } \quad \alpha, \beta, \gamma \in \mathrm{A}, \quad k, l \in \mathbb{I},
$$

где

$$
Q_{k l}^{\alpha \beta \gamma}=Q_{k l}^{\alpha \beta \gamma}(\Lambda)=R_{k l}^{\alpha \beta \gamma}+(-1)^{j}\left(\begin{array}{c}
j \\
p, k, l-i
\end{array}\right) D^{p} R_{i j}^{\beta \gamma \alpha}+(-1)^{i}\left(\begin{array}{c}
i \\
p, k-j, l
\end{array}\right) D^{p} R_{i j}^{\gamma \alpha \beta},
$$

$u\left(\begin{array}{c}j \\ p, k, l-i\end{array}\right),\left(\begin{array}{c}i \\ p, k-j, l\end{array}\right)$ суть триномиальнье коэффициентьы ${ }^{1)}$.

ДокАЗАтЕльство. Действительно, здесь

$$
\begin{aligned}
& R_{k l}^{\alpha \beta \gamma} \cdot D^{k} g_{\beta} \cdot D^{l} h_{\gamma}+(-D)^{l}\left(R_{k l}^{\beta \gamma \alpha} \cdot g_{\beta} \cdot D^{k} h_{\gamma}\right)+(-D)^{k}\left(R_{k l}^{\gamma \alpha \beta} \cdot D^{l} g_{\beta} \cdot h_{\gamma}\right)= \\
& =R_{k l}^{\alpha \beta \gamma} \cdot D^{k} g_{\beta} \cdot D^{l} h_{\gamma}+(-1)^{j}\left(\begin{array}{c}
j \\
p q r
\end{array}\right) D^{p} R_{k j}^{\beta \gamma \alpha} \cdot D^{q} g_{\beta} \cdot d^{r+k} h_{\gamma}+ \\
& \quad+(-1)^{i}\left(\begin{array}{c}
i \\
p q r
\end{array}\right) D^{p} R_{i l}^{\gamma \alpha \beta} \cdot D^{q+l} g_{\beta} \cdot D^{r} h_{\gamma}= \\
& =R_{k l}^{\alpha \beta \gamma} \cdot D^{k} g_{\beta} \cdot D^{l} h_{\gamma}+(-1)^{j}\left(\begin{array}{c}
j \\
p, k, l-i
\end{array}\right) D^{p} R_{i j}^{\beta \gamma \alpha} \cdot D^{k} g_{\beta} \cdot D^{l} h_{\gamma}+ \\
& \quad+(-1)^{i}\left(\begin{array}{c}
i \\
p, k-j, l
\end{array}\right) D^{p} R_{i j}^{\gamma \alpha \beta} \cdot D^{k} g_{\beta} \cdot D^{l} h_{\gamma}=Q_{k l}^{\alpha \beta \gamma} \cdot D^{k} g_{\beta} \cdot D^{l} h_{\gamma} .
\end{aligned}
$$

Для завершения доказательства достаточно воспользоваться предполагаемой горизонтальной точностью дифференциальной алгебры $\left(\mathcal{F}, \mathfrak{D}_{H}\right)$.

\footnotetext{
1)Напомним, полиномиальные коэффициенты $\left(\begin{array}{c}i \\ j_{1}, \ldots, j_{p}\end{array}\right)=\frac{i !}{j_{1} ! \ldots j_{p} !}, i=j_{1}+\cdots+j_{p}$. В частности, биномиальный коэффициент $\left(\begin{array}{c}i \\ j\end{array}\right)=\left(\begin{array}{c}i \\ j, i-j\end{array}\right)$.
} 


\section{6. ЭВОЛЮЦИЯ БЕЗ СВЯЗЕЙ}

Здесь (подробнее см. [1]) алгебра $\mathcal{F}=\mathcal{C}_{\text {fin }}^{\infty}(\mathrm{XU})$ есть алгебра всех гладких функций на бесконечномерном пространстве $\mathbf{X} \mathbf{U}=\mathbf{X} \times \mathbf{U}$, зависящих от конечного числа аргументов $x^{\mu}, u_{i}^{\alpha}$, где:

- $\mathrm{X}=\mathbb{R}^{\mathrm{M}}=\left\{x=\left(x^{\mu}\right) \mid x^{\mu} \in \mathbb{R}, \mu \in \mathrm{M}\right\}$ - пространство независимых переменных;

- $\mathrm{U}=\mathbb{R}^{\mathrm{A}}=\left\{u=\left(u^{\alpha}\right) \mid u^{\alpha} \in \mathbb{R}, \alpha \in \mathrm{A}\right\}$ - пространство зависимых переменных, $\mathrm{A}$ - конечное множество индексов;

- $\mathbf{U}=\mathbb{R}_{\mathbb{I}}^{\mathrm{A}}=\left\{\mathbf{u}=\left(u_{i}^{\alpha}\right) \mid u_{i}^{\alpha} \in \mathbb{R}, \alpha \in \mathrm{A}, i \in \mathbb{I}\right\}$ - пространство дифференциальных переменных, $u^{\alpha}=u_{0}^{\alpha}$.

Алгебра Ли $\mathfrak{D}_{H}$ имеет $\mathcal{F}$-базис $D=\left\{D_{\mu} \mid \mu \in \mathrm{M}\right\}$, где полные частные производные

$$
D_{\mu}=\partial_{x^{\mu}}+u_{i+(\mu)}^{\alpha} \partial_{u_{i}^{\alpha}}, \quad i+(\mu)=\left(i^{1}, \ldots, i^{\mu}+1, \ldots, i^{m}\right) .
$$

Подалгебра Ли $\mathfrak{D}_{V}$ имеет $\mathcal{F}$-базис $\left\{\partial_{u_{i}^{\alpha}} \mid \alpha \in \mathrm{A}, i \in \mathbb{I}\right\}$, так что здесь $a=\left(\begin{array}{c}\alpha \\ i\end{array}\right)$, $\mathbf{A}=\left(\begin{array}{c}\mathrm{A} \\ \mathbb{I}\end{array}\right)$, и коммутатор

$$
\left[D_{\mu}, \partial_{u_{i}^{\alpha}}\right]=-\partial_{u_{i-(\mu)}^{\alpha}}, \quad \text { т. е. } \quad \Gamma_{\mu \alpha j}^{i \beta}=-\delta_{\alpha}^{\beta} \delta_{j+(\mu)}^{i}, \quad \alpha, \beta \in \mathrm{A}, \quad i, j \in \mathbb{I}, \quad \mu \in \mathrm{M} .
$$

Отметим, что здесь пара $\left(\begin{array}{c}\beta \\ j\end{array}\right)$ есть единый верхний индекс, а пара $\left(\begin{array}{c}i \\ \alpha\end{array}\right)$ есть единый нижний индекс.

Далее, в этой ситуации,

- $\nabla: \mathcal{F}_{\mathbb{I}}^{\mathrm{A}} \rightarrow \mathcal{F}_{\mathrm{MI}}^{\mathrm{A}}, \quad \phi=\left(\phi_{i}^{\alpha}\right) \mapsto \eta=\left(\eta_{\mu i}^{\alpha}\right), \quad \eta_{\mu i}^{\alpha}=D_{\mu} \phi_{i}^{\alpha}-\phi_{i+(\mu)}^{\alpha} ;$

- $\mathbf{j}: \mathcal{F}^{\mathrm{A}} \rightarrow \mathcal{F}_{\mathbb{I}}^{\mathrm{A}}, \quad \phi=\left(\phi^{\alpha}\right) \mapsto \phi=\left(\phi_{i}^{\alpha}\right), \quad \phi_{i}^{\alpha}=D^{i} \phi^{\alpha}=\delta_{i k} \delta_{\beta}^{\alpha} \cdot D^{k} \phi^{\beta}$;

- $\operatorname{Ker} \mathbf{j}=0, \quad \operatorname{Im} \mathbf{j}=\operatorname{Ker} \nabla, \quad\left[\mathrm{ev}_{\boldsymbol{\phi}}, \mathbf{j}\right]=0$ для всех $\boldsymbol{\phi} \in \mathcal{E}$;

- $Q_{k l}^{\alpha \beta \gamma}=R_{k l}^{\alpha \beta \gamma}+(-1)^{j}\left(\begin{array}{c}j \\ p, k, l-i\end{array}\right) D^{p} R_{i j}^{\beta \gamma \alpha}+(-1)^{i}\left(_{p, k-j, l}^{i}\right) D^{p} R_{i j}^{\gamma \alpha \beta}$;

- $R_{k l}^{\alpha \beta \gamma}=\left(\begin{array}{c}i \\ r\end{array}\right) D^{i-r} \Lambda_{k-r}^{\epsilon \beta} \cdot \partial_{u_{i}^{\epsilon}} \Lambda_{l}^{\alpha \gamma}, \quad \alpha, \beta, \gamma \in \mathrm{A}, \quad k, l, i, r, j, p \in \mathbb{I}$.

\section{7. ПРОСТЕЙШИЙ СЛУЧАЙ}

Здесь мы считаем, что

- $\mathrm{X}=\mathbb{R}, \quad \mathrm{U}=\mathbb{R}, \quad \mathrm{U}=\mathbb{R}_{\mathbb{I}}, \quad \mathbb{I}=\mathbb{Z}_{+}, \quad \mathcal{F}=\mathcal{C}_{\text {fin }}^{\infty}(\mathrm{XU})$

- $D=\partial_{x}+u_{i+1} \partial_{u_{i}}, \quad\left[D, \partial_{u_{i}}\right]=-\partial_{u_{i-1}}, \quad i \in \mathbb{I}$.

В этом случае горизонтальный дифференциальный оператор $\Lambda(D)=\sum_{i=0}^{s} \Lambda_{i} \cdot D^{i}$, где порядок $s \in \mathbb{N}$, коэффициенты $\Lambda_{i}=\Lambda_{i}\left(u_{0}, u_{1}, \ldots, u_{n(i)}\right) \in \mathcal{F}, 0 \leqslant i \leqslant s$. В частности (см. лемму 2, теорему 1 ),

- $R_{k l}=\left(\begin{array}{c}i \\ r\end{array}\right) D^{i-r} \Lambda_{k-r} \cdot \partial_{u_{i}} \Lambda_{l}, \quad k, l, i, r, j, p \in \mathbb{I}$

- $Q_{k l}=R_{k l}+(-1)^{j}\left(\begin{array}{c}j \\ p, k, l-i\end{array}\right) D^{p} R_{i j}+(-1)^{i}\left(_{p, k-j, l}^{i}\right) D^{p} R_{i j}$;

- $\operatorname{supp} R=\left\{(k, l) \in \mathbb{I}^{2} \mid l \leqslant s, k \leqslant n(l)+s\right\}$;

- $\operatorname{sp} R_{k l}=\left\{(r, i) \in \mathbb{I}^{2} \mid \max \{0, k-s\} \leqslant r \leqslant k, r \leqslant i \leqslant n(l)\right\}$.

ПРЕДЛОЖЕНИЕ 5. В этих обозначениях

$$
R_{k l}=\sum_{s p R_{k l}}\left(\begin{array}{l}
i \\
r
\end{array}\right) D^{i-r} \Lambda_{k-r} \cdot \partial_{u_{i}} \Lambda_{l}, \quad(k, l) \in \operatorname{supp} R,
$$

$u R_{k l}=0$ для $(k, l) \notin \operatorname{supp} R$. 


\section{Положим}

$$
Q_{k l}=R_{k l}+Q_{k l}^{\prime}+Q_{k l}^{\prime \prime}, \quad(k, l) \in \operatorname{supp} Q,
$$

где $\operatorname{supp} Q=\operatorname{supp} R \cup \operatorname{supp} Q^{\prime} \cup \operatorname{supp} Q^{\prime \prime} \subset \mathbb{I}^{2}$,

- $\operatorname{supp} Q^{\prime}=\bigcup_{(i, j) \in \operatorname{supp} R}\left\{(k, l) \in \mathbb{I}^{2} \mid k \leqslant j, l \geqslant i, k+l \leqslant i+j\right\}$,

- $\operatorname{supp} Q^{\prime \prime}=\bigcup_{(i, j) \in \operatorname{supp} R}\left\{(k, l) \in \mathbb{I}^{2} \mid k \geqslant j, l \leqslant i, k+l \leqslant i+j\right\}$.

По определению $R_{k l}=0$ для $(k, l) \notin \operatorname{supp} R, Q_{k l}^{\sharp}=0$ для $(k, l) \notin \operatorname{supp} Q^{\sharp}$, где $\sharp={ }^{\prime},{ }^{\prime}$.

7.1. Пример. Рассмотрим оператор $\Lambda(D)=\lambda D+D \lambda / 2, \lambda=\lambda\left(u_{0}, \ldots, u_{n}\right) \in \mathcal{F}$, $n \in \mathbb{Z}_{+}$. Здесь $s=1, \Lambda_{0}=D \lambda / 2, \Lambda_{1}=\lambda, n(i)=n+1-i$ при $i=0,1$, так что $n(i)+i=n+1$. По построению оператор $\Lambda(D)$ кососимметрический по Лагранжу. В силу теоремы 1 , функция $\lambda$ определяется системой дифференциальных уравнений $Q_{k l}=Q_{k l}(\lambda)=0, k, l \in \mathbb{I}$.

Вычисления показывают, что supp $R=\left\{(k, l) \in \mathbb{I}^{2} \mid l \leqslant 1, k \leqslant n(l)+1\right\}$.

Обозначим $\lambda_{u_{i}}=\partial_{u_{i}} \lambda, i=0, \ldots, n$, и положим

$$
\begin{aligned}
\left(\begin{array}{l}
i \\
k
\end{array}\right)^{\prime} & =\left(\begin{array}{l}
i \\
k
\end{array}\right)+2\left(\begin{array}{c}
i \\
k-1
\end{array}\right), \\
\left(\begin{array}{c}
i \\
p k l
\end{array}\right)^{\prime} & =\left(\begin{array}{c}
i \\
p, k-1, l
\end{array}\right)-\left(\begin{array}{c}
i \\
p, k, l-1
\end{array}\right)=\frac{(k-l) i !}{p ! k ! l !}, \quad p+k+l=i+1 .
\end{aligned}
$$

Итак (напомним, что мы предполагаем суммирование по повторяющимся верхним и нижним индексам в естественных пределах),

$$
\begin{aligned}
R_{k l} & =\left(\begin{array}{c}
i \\
r
\end{array}\right) D^{i-r} \Lambda_{k-r} \cdot \partial_{u_{i}} \Lambda_{l}=(k-r=0,1)= \\
& =\left(\left(\begin{array}{l}
i \\
k
\end{array}\right) D^{i-k} \Lambda_{0}+\left(\begin{array}{c}
i \\
k-1
\end{array}\right) D^{i+1-k} \Lambda_{1}\right) \cdot \partial_{u_{i}} \Lambda_{l}= \\
& =\frac{1}{2}\left(\left(\begin{array}{l}
i \\
k
\end{array}\right) D^{i+1-k} \lambda+2\left(\begin{array}{c}
i \\
k-1
\end{array}\right) D^{i+1-k} \lambda\right) \cdot \partial_{u_{i}} \Lambda_{l}=\frac{1}{2}\left(\begin{array}{c}
i \\
k
\end{array}\right)^{\prime} D^{i+1-k} \lambda \cdot \partial_{u_{i}} \Lambda_{l} .
\end{aligned}
$$

В частности,

$$
R_{k 1}=\frac{1}{2}\left(\begin{array}{c}
i \\
k
\end{array}\right)^{\prime} D^{i+1-k} \lambda \cdot \lambda_{u_{i}}, \quad k=0, \ldots, n+1
$$

тогда как

$$
\begin{aligned}
R_{k 0} & =\frac{1}{4}\left(\begin{array}{c}
i \\
k
\end{array}\right)^{\prime} D^{i+1-k} \lambda \cdot \partial_{u_{i}}(D \lambda)=\left(\partial_{u_{i}} \circ D=D \circ \partial_{u_{i}}+\partial_{u_{i-1}}\right)= \\
& =\frac{1}{4}\left(\begin{array}{c}
i \\
k
\end{array}\right)^{\prime} D^{i+1-k} \lambda \cdot D \lambda_{u_{i}}+\left(\begin{array}{l}
i \\
k
\end{array}\right)^{\prime} D^{i+1-k} \lambda \cdot \lambda_{u_{i-1}}= \\
& =\left(\left(\begin{array}{c}
i+1 \\
k
\end{array}\right)^{\prime}-\left(\begin{array}{c}
i \\
k
\end{array}\right)^{\prime}=\left(\begin{array}{c}
i \\
k-1
\end{array}\right)^{\prime}\right)= \\
& =\frac{1}{4}\left(\left(\begin{array}{c}
i \\
k
\end{array}\right)^{\prime}\left(D^{i+2-k} \lambda \cdot \lambda_{u_{i}}+D^{i+1-k} \lambda \cdot D \lambda_{u_{i}}\right)+\left(\begin{array}{c}
i \\
k-1
\end{array}\right)^{\prime} D^{i+2-k)} \lambda \cdot \lambda_{u_{i}}\right)= \\
& =\frac{1}{4}\left(D\left(\left(\begin{array}{c}
i \\
k
\end{array}\right)^{\prime} D^{i+1-k} \lambda \cdot \lambda_{u_{i}}\right)+\left(\begin{array}{c}
i \\
k-1
\end{array}\right)^{\prime} D^{i+1-(k-1)} \lambda \cdot \lambda_{u_{i}}\right),
\end{aligned}
$$


так что

$$
R_{k 0}=\frac{1}{2}\left(D R_{k 1}+R_{k-1,1}\right), \quad k=0, \ldots, n+2
$$

Следовательно,

$$
\begin{aligned}
Q_{k l}^{\prime} & =(-1)^{j}\left(\begin{array}{c}
j \\
p, k, l-i
\end{array}\right) D^{p} R_{i j}=(j=0,1)= \\
& =\left(\begin{array}{c}
0 \\
p, k, l-i
\end{array}\right) D^{p} R_{i 0}-\left(\begin{array}{c}
1 \\
p, k, l-i
\end{array}\right) D^{p} R_{i 1}= \\
& =\delta_{k}^{0} R_{l 0}-\delta_{k}^{0} D R_{l 1}-\delta_{k+(l-i)}^{1} R_{i 1}=\left(D R_{l 1}+R_{l-1,1}=2 R_{l 0}\right)= \\
& =-\left(\delta_{k}^{0} R_{l 0}+\delta_{k}^{1} R_{l 1}\right)=-R_{l k}
\end{aligned}
$$

т. e.

$$
Q_{k l}^{\prime}=-R_{l k}, \quad l=0, \ldots, n+2, \quad k=0,1 .
$$

Далее,

$$
\begin{aligned}
Q_{k l}^{\prime \prime} & =(-1)^{i}\left(\begin{array}{c}
i \\
p, k-j, l
\end{array}\right) D^{p} R_{i j}=(j=0,1)= \\
& =(-1)^{i}\left(\begin{array}{c}
i \\
p k l
\end{array}\right) D^{p} R_{i 0}+(-1)^{i}\left(\begin{array}{c}
i \\
p, k-1, l
\end{array}\right) D^{p} R_{i 1}=\left(2 R_{i 0}=D R_{i 1}+R_{i-1,1}\right)= \\
& =\frac{1}{2}(-1)^{i}\left(\begin{array}{c}
i \\
p k l
\end{array}\right) D^{p}\left(D R_{i 1}+R_{i-1,1}\right)+(-1)^{i}\left(\begin{array}{c}
i \\
p, k-1, l
\end{array}\right) D^{p} R_{i 1}= \\
& =\frac{1}{2}(-1)^{i}\left(\left(\begin{array}{c}
i \\
p-1, k, l
\end{array}\right)-\left(\begin{array}{c}
i+1 \\
p k l
\end{array}\right)+2\left(\begin{array}{c}
i \\
p, k-1, l
\end{array}\right)\right) D^{p} R_{i 1}= \\
& =\frac{1}{2}(-1)^{i}\left(\left(\begin{array}{c}
i \\
p, k-1, l
\end{array}\right)-\left(\begin{array}{c}
i \\
p, k, l-1
\end{array}\right)\right) D^{p} R_{i 1},
\end{aligned}
$$

где мы воспользовались равенством

$$
\left(\begin{array}{c}
i \\
p-1, k, l
\end{array}\right)+\left(\begin{array}{c}
i \\
p, k-1, l
\end{array}\right)+\left(\begin{array}{c}
i \\
p, k, l-1
\end{array}\right)=\left(\begin{array}{c}
i+1 \\
p k l
\end{array}\right), \quad p+k+l=i+1 .
$$

Так что

$$
Q_{k l}^{\prime \prime}=\frac{(-1)^{i}}{2}\left(\begin{array}{c}
i \\
p k l
\end{array}\right)^{\prime} D^{p} R_{i 1}, \quad i=0, \ldots, n+1, \quad p+k+l=i+1 \leqslant n+2 .
$$

Заметим, что $Q_{k l}^{\prime \prime}=-Q_{l k}^{\prime \prime}$ в силу равенства $\left(\begin{array}{c}i \\ p k l\end{array}\right)^{\prime}=-\left(\begin{array}{c}i \\ p l k\end{array}\right)^{\prime}($ см. определение $(1))$.

Итак,

$$
Q_{k l}=Q_{k l}(\lambda)=R_{k l}-R_{l k}+\frac{(-1)^{i}}{2}\left(\begin{array}{c}
i \\
p k l
\end{array}\right)^{\prime} D^{p} R_{i 1}=-Q_{l k} .
$$

Наша цель теперь - найти все функции $\lambda=\lambda\left(u_{0}, \ldots, u_{n}\right) \in \mathcal{F}, n \in \mathbb{Z}_{+}$, такие, что $Q(\lambda)=\left(Q_{k l}(\lambda)\right)=0$. Удобно начать с уравнения $Q_{1, n+1}=0, n \in \mathbb{N}$. Здесь, $R_{1, n+1}=0, R_{n+1,1}=\lambda \cdot \lambda_{u_{n}}$, тогда как

$$
Q_{1, n+1}^{\prime \prime}=\frac{(-1)^{i}}{2}\left(\begin{array}{c}
i \\
p, 1, n+1
\end{array}\right)^{\prime} D^{p} R_{i 1}=\frac{(-1)^{n+1}}{2}\left(\begin{array}{c}
n+1 \\
0,1, n+1
\end{array}\right)^{\prime} R_{n+1,1}=\frac{(-1)^{n} n}{2} R_{n+1,1} .
$$


Таким образом,

$$
Q_{1, n+1}=-R_{n+1,1}+\frac{(-1)^{n} n}{2} R_{n+1,1}=\left(\frac{(-1)^{n} n}{2}-1\right) R_{n+1,1} \begin{cases}=0, & n=2 \\ \neq 0, & n>2\end{cases}
$$

Следовательно, уравнение $Q_{1, n+1}=0$ влечет $\partial_{u_{n}} \lambda=0$ при $n>2$, другими словами, $\lambda=\lambda\left(u_{0}, u_{1}, u_{2}\right)$. Случай $n=2$ требует дальнейшего детального рассмотрения. Именно, здесь

$$
\begin{aligned}
& Q_{04}=0-R_{40}+\frac{(-1)^{2}}{2} R_{31}=-\frac{1}{2} R_{31}+\frac{(-1)^{2}}{2} R_{31}=0, \\
& Q_{03}=0-R_{30}-\frac{1}{2} R_{21}+\frac{3}{2} D R_{31}=D R_{31}-R_{21}, \\
& Q_{02}=0-R_{20}+\frac{1}{2}\left(3 D^{2} R_{31}-2 D R_{21}+R_{11}\right)=\frac{3}{2} D\left(D R_{31}-R_{21}\right)=\frac{3}{2} D Q_{03}, \\
& Q_{01}=R_{01}-R_{10}+\frac{1}{2}\left(D^{3} R_{31}-D^{2} R_{21}+D R_{11}-R_{01}\right)=\frac{1}{2} D^{2} Q_{03}, \\
& Q_{13}=0-R_{31}+R_{31}=0, \\
& Q_{12}=0-R_{21}+\left(\frac{3}{2} D R_{31}-\frac{1}{2} R_{21}\right)=\frac{3}{2} Q_{03},
\end{aligned}
$$

где мы воспользовались формулами (3) и (6). Все остальные уравнения несущественны. Таким образом, единственным существенным уравнением является следующее уравнение: $Q_{03}=D R_{31}-R_{21}=0$. Здесь

$$
\begin{aligned}
& R_{31}=\lambda \cdot \lambda_{u_{2}}, \quad D R_{31}=D \lambda \cdot \lambda_{u_{2}}+\lambda \cdot D \lambda_{u_{2}}, \quad R_{21}=\lambda \cdot \lambda_{u_{1}}+\frac{5}{2} D \lambda \cdot \lambda_{u_{2}} \\
& Q_{03}=\lambda \cdot D \lambda_{u_{2}}-\frac{3}{2} D \lambda \cdot \lambda_{u_{2}}-\lambda \cdot \lambda_{u_{1}}=u_{3}\left(\lambda \cdot \lambda_{u_{2} u_{2}}-\frac{3}{2} \lambda_{u_{2}}^{2}\right)+\tilde{Q}_{03}\left(u_{0}, u_{1}, u_{2}\right) .
\end{aligned}
$$

Уравнение $\lambda \cdot \lambda_{u_{2} u_{2}}-3 \lambda_{u_{2}}^{2} / 2=0$ имеет общее решение $\lambda=\left(\phi u_{2}+\psi\right)^{-2}$, функции $\phi, \psi\left(u_{0}, u_{1}\right) \in \mathcal{F}$ произвольные. Приведенное уравнение имеет вид

$$
\tilde{Q}_{03}=\lambda \cdot\left(\lambda_{u_{1} u_{2}} u_{2}+\lambda_{u_{0} u_{2}} u_{1}\right)-\frac{3}{2}\left(\lambda_{u_{1}} u_{2}+\lambda_{u_{0}} u_{1}\right) \cdot \lambda_{u_{2}}-\lambda \cdot \lambda_{u_{1}}=0,
$$

где

$$
\begin{aligned}
& \lambda_{u_{i}}=-2\left(\phi u_{2}+\psi\right)^{-3}\left(\phi_{u_{i}} u_{2}+\psi_{u_{i}}\right), \quad i=0,1, \quad \lambda_{u_{2}}=-2\left(\phi u_{2}+\psi\right)^{-3} \phi \\
& \lambda_{u_{i} u_{2}}=2\left(\phi u_{2}+\psi\right)^{-4}\left(3\left(\phi_{u_{i}} u_{2}+\psi_{u_{i}}\right) \phi-\left(\phi u_{2}+\psi\right) \phi_{u_{i}}\right), \quad i=0,1 .
\end{aligned}
$$

После подстановки и ряда простых преобразований получаем окончательную форму этого уравнения:

$$
\tilde{Q}_{03}=2\left(\phi u_{2}+\psi\right)^{-5}\left(\psi_{u_{1}}-\phi_{u_{0}} u_{1}\right)=0 .
$$

Результирующее уравнение $\psi_{u_{1}}-\phi_{u_{0}} u_{1}=0$ имеет общее решение

$$
\phi\left(u_{0}, u_{1}\right)=\left.\chi_{v}(u, v)\right|_{u=u_{0}, v=u_{1}^{2} / 2}, \quad \psi\left(u_{0}, u_{1}\right)=\left.\chi_{u}(u, v)\right|_{u=u_{0}, v=u_{1}^{2} / 2},
$$

где $\chi(u, v) \in \mathcal{C}^{\infty}\left(\mathbb{R}^{2}\right)$ есть произвольная гладкая функция.

Мы суммируем наши вычисления в виде следующей теоремы. 
Теорема 2 (ср. [7], [8]). Дифференциальный оператор $\Lambda(D)=\lambda D+D \lambda / 2, \lambda \in \mathcal{F}$, является гамильтоновым тогда и только тогда, когда $\lambda=\left(\phi u_{2}+\psi\right)^{-2}$, где функици $\phi, \psi\left(u_{0}, u_{1}\right) \in \mathcal{F}$ заданъь формулами (7).

\section{Список литературы}

[1] В.В. Жаринов, "Структуры Ли-Пуассона над дифференциальными алгебрами", $T M \Phi$, 192:3 (2017), 459-472.

[2] П. Олвер, Приложения групп Ли к дифференциальным уравнениям, Мир, М., 1989.

[3] V.V. Zharinov, Lecture Notes on Geometrical Aspects of Partial Differential Equations, World Sci., Singapore, 1992.

[4] Н. Х. Ибрагимов, Группы преобразований в математической физике, Наука, М., 1983.

[5] В. В. Жаринов, "Эволюционные системы со связями в виде условий нулевой дивергенции", ТМФ, 163:1 (2010), 3-16.

[6] В. В. Жаринов, “О преобразовании Беклунда", ТМФ, 189:3 (2016), 323-334.

[7] И. М. Гельфанд, И. Я. Дорфман, "Гамильтоновы операторы и связанные с ними алгебраические структуры”, Функи. анализ и его прил., 13:4 (1979), 13-30.

[8] Б. А. Дубровин, С.П. Новиков, "Гидродинамика слабо деформированных солитонных решеток. Дифференциальная геометрия и гамильтонова теория", УМH, 44:6(270) (1989), 29-98.

[9] М. О. Катанаев, "Векторные поля Киллинга и однородная и изотропная вселенная", УФH, 186:7 (2016), 763-775.

[10] G. A. Alekseev, "Collision of strong gravitational and electromagnetic waves in the expanding universe", Phys. Rev. D, 93:6 (2016), 061501, 6 pp.

[11] А.П. Чугайнова, В.А. Шаргатов, "Устойчивость структуры разрывов, описываемых обобщенным уравнением Кортевега-д Вриза-Бюргерса", Ж. вычисл. матем. и матем. физ., 56:2 (2016), 259-274.

[12] А.Г. Куликовский, А.П. Чугайнова, "Исследование разрывов в решениях уравнений упругопластической среды Прандтля-Рейсса", Ж. вычисл. матем. и матем. физ., $\mathbf{5 6 : 4}(2016), 650-663$.

[13] В. А. Васильев, “Локальные лакуны Петровского вблизи параболических особенностей волновых фронтов строго гиперболических уравнений в частных производных", $M a-$ тем. сб., 207:10 (2016), 4-27.

[14] В. В. Козлов, “Об уравнениях гидродинамического типа”, ПММ, 80:3 (2016), 294-303.

[15] В. М. Бухштабер, "Полиномиальные динамические системы и уравнение Кортевега-де Фриза", Тр. МИАН, 294 (2016), 191-215.

[16] В.П. Павлов, В. М. Сергеев, "Гидродинамика и термодинамика как единая полевая теория", Тр. МИАН, 294 (2016), 237-247.

[17] А. Г. Куликовский, А. П. Чугайнова, “Автомодельная задача о волнах в упругопластической среде Прандтля-Рейсса", Тр. МИАН, 295 (2016), 195-205.

[18] А. Т. Ильичев, А. П. Чугайнова, "Теория спектральной устойчивости гетероклинических решений уравнения Кортевега-де Фриза-Бюргерса с произвольным потенциалом", Тр. МИАН, 295 (2016), 163-173.

[19] Г. А. Алексеев, “Интегрируемые и неинтегрируемые структуры в уравнениях Эйнштейна-Максвелла с абелевой группой изометрий G2G2”, Тр. МИАН, 295 (2016), 7-33. 